\title{
One-Dimensional versus Three-Dimensional Approaches to the Rydberg Wave Packet Dynamics
}

\author{
T. KOPYCIUK* AND R. PARZYŃSKI \\ Faculty of Physics, A. Mickiewicz University \\ Umultowska 85, 61-614 Poznań, Poland
}

(Received February 13, 2006; revised version May 8, 2006)

\begin{abstract}
We show that a one-dimensional approximation to a real three-dimensional atom offers good results for a selected group of the Rydberg states. It is demonstrated in the context of evolution of the Rydberg wave packets produced by the so-called half-cycle pulses.
\end{abstract}

PACS numbers: 32.80.Rm, 03.65.Ge

\section{Introduction}

In recent years there has been increasing interest in the generation and control of the Rydberg wave packets because of their possible use for, e.g., data storage and processing [1]. Such operations can be performed by the application of a single unidirectional half-cycle electric-field pulse (HCP) [2] of a duration much shorter than the Kepler period $T_{n}=2 \pi n^{3}$ (in atomic units, a.u.) of the $n$-th Rydberg state $(n \gg 1)$. HCPs are used for creating, manipulating and also sampling of the Rydberg wave packets [3]. However, the dynamics of the Rydberg atoms kicked by HCP is not well understood yet and it is often hard to analyze it because of inconvenient formulae for the real three-dimensional (3D) atom. This problem can be simplified, fortunately, when it is justified to introduce a one-dimensional (1D) model of the Rydberg atom. Such a nearly 1D atom is, in practice, created by photoexcitation of extreme members of the Stark manifolds in the presence of a weak dc electric field [4]. The aim of this paper is to present a description of the 1D Rydberg wave packet dynamics and to evaluate the correctness of this approach by comparing the $1 \mathrm{D}$ results with the $3 \mathrm{D}$ ones.

\section{1D versus $3 \mathrm{D}$ Rydberg atoms}

The 1D Rydberg atom is described by the Hamiltonian (in a.u.) [5]:

$H=-\frac{1}{2} \frac{\mathrm{d}^{2}}{\mathrm{~d} x^{2}}-\frac{1}{x}, \quad x>0$

${ }^{*}$ corresponding author; e-mail: a100@interia.pl 
of the eigenvalues

$$
E_{n}=-\frac{1}{2 n^{2}}
$$

and eigenfunctions

$$
|n\rangle=\frac{2 x}{\sqrt{n^{3}}} \mathrm{e}^{-x / n} F(1-n, 2,2 x / n),
$$

where $F(a, b, c)$ is the confluent hypergeometric function. The use of the Fourier transform results in the following momentum-space eigenfunction for the $1 \mathrm{D}$ Rydberg atom [6]:

$$
|n(p)\rangle=\sqrt{2 n / \pi} \frac{(n p+\mathrm{i})^{n-1}}{(n p-\mathrm{i})^{n+1}} .
$$

Because the classical trajectory of the 1D Rydberg atom mimics a line on one side of the atom's core, the compatibility between the 1D and 3D cases should be the greatest when the probability density calculated from the $3 \mathrm{D}$ wave function changes in one dimension, predominantly. The wave function of the $3 \mathrm{D}$ hydrogen atom looks, in the parabolic coordinates, like [7]

$$
\begin{aligned}
& \Psi_{n, n_{1}, n_{2}, m}=\frac{1}{\sqrt{\pi} n^{2}} \frac{1}{n^{|m|}(|m| !)^{2}} \sqrt{\frac{\left(n_{1}+|m|\right) !\left(n_{2}+|m|\right) !}{n_{1} ! n_{2} !}} \\
& \times \mathrm{e}^{\mathrm{i} m \varphi} \xi^{\frac{|m|}{2}} \mathrm{e}^{-\frac{\xi}{2 n}} F\left(-n_{1},|m|+1, \frac{\xi}{n}\right) \eta^{\frac{|m|}{2}} \mathrm{e}^{-\frac{\eta}{2 n}} F\left(-n_{2},|m|+1, \frac{\eta}{n}\right) .
\end{aligned}
$$

We choose to use the parabolic coordinates because they are the most convenient when working with the Rydberg states from different ends of the Stark spectrum. Here, $n_{1}$ and $n_{2}$ are the parabolic quantum numbers fulfilling the relation $n_{1}+$ $n_{2}+|m|+1=n$.

For $n$ increasing from 6 to 60, we show in Table the ratio of the expectation value of $z$ to the expectation value of $\rho=\sqrt{x^{2}+y^{2}}$ for the extreme Stark states,

\section{TABLE}

Ratios of $\bar{z} / \bar{\rho}$, where $\rho=\sqrt{x^{2}+y^{2}}$, for the red $\left(n_{1}=0, n_{2}=n-1\right)$, blue $\left(n_{1}=n-1, n_{2}=0\right)$ and intermediate $\left(n_{1}=n / 2, n_{2}=n / 2-1\right)$ Stark states versus $n$.

\begin{tabular}{c|c|c|c}
\hline \hline$n$ & $\bar{z} / \bar{\rho}$ (red) & $\bar{z} / \bar{\rho}$ (intermediate) & $\bar{z} / \bar{\rho}$ (blue) \\
\hline 6 & -2.102 & 0.234 & 2.102 \\
10 & -2.892 & 0.139 & 2.892 \\
20 & -4.276 & 0.069 & 4.276 \\
30 & -5.312 & 0.046 & 5.312 \\
40 & -6.177 & 0.035 & 6.177 \\
50 & -6.935 & 0.028 & 6.935 \\
60 & -7.618 & 0.023 & 7.618
\end{tabular}


i.e., the red $\left(n_{1}=0, n_{2}=n-1\right)$ and blue $\left(n_{1}=n-1, n_{2}=0\right)$ ones and also for an intermediate Stark state ( $n_{1}$ comparable to $\left.n_{2}\right)$. The ratio is a measure of quality of the 1D approximation and the higher ratio the better approximation. As we can see, this ratio can be high for extreme Stark states, while for a state from the center of the Stark manifold it is very small. The ratios for the extreme (blue and red) Stark states are seen to increase with increasing $n$. We thus expect that a reasonably large $n$ is needed for the $1 \mathrm{D}$ approximation to be justified for extreme Stark states.

\section{Rydberg wave packet dynamics}

When describing the Rydberg packet dynamics the most important is the so-called form factor, which is the transition amplitude between any two Rydberg states in the impulse approximation [8]. The form factor between f-th and i-th state is defined as $T_{\mathrm{fi}}\left\langle\mathrm{f}\left|\mathrm{e}^{\mathrm{i} q x}\right| \mathrm{i}\right\rangle$, where $q$ is a momentum (in a.u.) transferred to the atom from HCP. For the 1D case, it was derived by Bersons and Veilande [5], although their formula needs to be multiplied by $n n^{\prime}$ :

$$
\begin{aligned}
T_{n n^{\prime}} & =-\frac{z(\lambda-2 / n)^{n}\left(\lambda-2 / n^{\prime}\right)^{n^{\prime}}}{\sqrt{n n^{\prime}} \lambda^{n+n^{\prime}}}\left[\left(\frac{n-1}{\lambda-2 / n}+\frac{n^{\prime}-1}{\lambda-2 / n^{\prime}}-\frac{n+n^{\prime}}{\lambda}\right)\right. \\
& \times F\left(1-n, 1-n^{\prime}, 2, z\right) F\left(1-n, 1-n^{\prime}, 2, z\right) \\
& \left.-\frac{(n-1)\left(n^{\prime}-1\right)}{2}\left(\frac{1}{\lambda-2 / n}+\frac{1}{\lambda-2 / n^{\prime}}\right) z F\left(2-n, 2-n^{\prime \prime}, 3, z\right)\right],
\end{aligned}
$$

where

$$
\lambda=\frac{1}{n}+\frac{1}{n^{\prime}}-\mathrm{i} q, \quad z=-\frac{4 n n^{\prime}}{\left(n-n^{\prime}\right)^{2}+y^{2}}, \quad y=q n n^{\prime}
$$

and $F(a, b, c, z)$ is the hypergeometric function. For the 3D case the form factor is given by [9]:

$$
\begin{aligned}
T_{\mathrm{fi}}= & \mathrm{i} y z \frac{2^{2|m|+1}\left(n n^{\prime}\right)|m|}{(|m| !)^{2}} \sqrt{\frac{\left(n_{1}+|m|\right) !\left(n_{1}^{\prime}+|m|\right) !\left(n_{2}+|m|\right) !\left(n_{2}^{\prime}+|m|\right) !}{n_{1} ! n_{1}^{\prime} ! n_{2} ! n_{2}^{\prime} !}} \\
& \times \frac{\left(n-n^{\prime}-\mathrm{i} y\right)^{n_{1}}\left(n^{\prime}-n-\mathrm{i} y\right)^{n_{1}^{\prime}}\left(n-n^{\prime}+\mathrm{i} y\right)^{n_{2}}\left(n^{\prime}-n+\mathrm{i} y\right)^{n_{2}^{\prime}}}{\left(n+n^{\prime}-\mathrm{i} y\right)^{n_{1}+n_{1}^{\prime}+|m|+1}\left(n+n^{\prime}+\mathrm{i} y\right)^{n_{2}+n_{2}^{\prime}+|m|+1}} \\
& \times\left\{\frac{2 n n^{\prime}\left(n_{1}-n_{2}\right)+\left(n_{2}^{\prime}-n_{1}^{\prime}\right)\left(n^{2}+n^{\prime 2}+y^{2}\right)}{\left(n+n^{\prime}\right)^{2}+y^{2}}\right. \\
& \times F\left[-n_{1},-n_{1}^{\prime},|m|+1, z(y=0)\right] F\left[-n_{2},-n_{2}^{\prime},|m|+1, z(y=0)\right] \\
& +n_{1}^{\prime} F\left[-n_{1},-n_{1}^{\prime}+1,|m|+1, z(y=0)\right] F\left[-n_{2},-n_{2}^{\prime},|m|+1, z(y=0)\right] \\
-n_{2}^{\prime} F[- & \left.\left.n_{1},-n_{1}^{\prime},|m|+1, z(y=0)\right] F\left[-n_{2},-n_{2}^{\prime}+1,|m|+1, z(y=0)\right]\right\}
\end{aligned}
$$


Both Eq. (6) and Eq. (8) were rederived by us along a different line and we confirm their correctness.

In Fig. 1 we present the $1 \mathrm{D}$ and 3D form factors of survival of the atom in its initial state after the action of HCP. Because of a complex character of the form factors, there are shown their real and imaginary parts, separately, versus the scaled momentum transfer. The $3 \mathrm{D}$ form factors have been calculated for the extreme blue Stark states $\left(\left|n, n_{1}, n_{2},\right| m|\rangle=|10,9,0,0\rangle,|30,29,0,0\rangle\right.$ and $|80,79,0,0\rangle$, respectively), for which the $1 \mathrm{D}$ approximation is expected to be valid (see Table). The results for the extreme red Stark states $\left(\left|n, n_{1}, n_{2},\right| m|\rangle=\right.$ $|10,0,9,0\rangle,|30,0,29,0\rangle)$ and $|80,0,79,0\rangle)$ are exactly the same. As seen from Fig. 1, the agreement between the $1 \mathrm{D}$ and $3 \mathrm{D}$ calculations is very good at low $q$, but some discrepancies appear with increasing $q$ if $n$ is not reasonably large. However, these discrepancies vanish for increasing $n$, as seen from the middle and bottom rows of Fig. 1. As seen from Fig. 2, the 1D approximation (working well for the extreme Stark states) completely loses its validity in the case of the middle states from the Stark manifold.
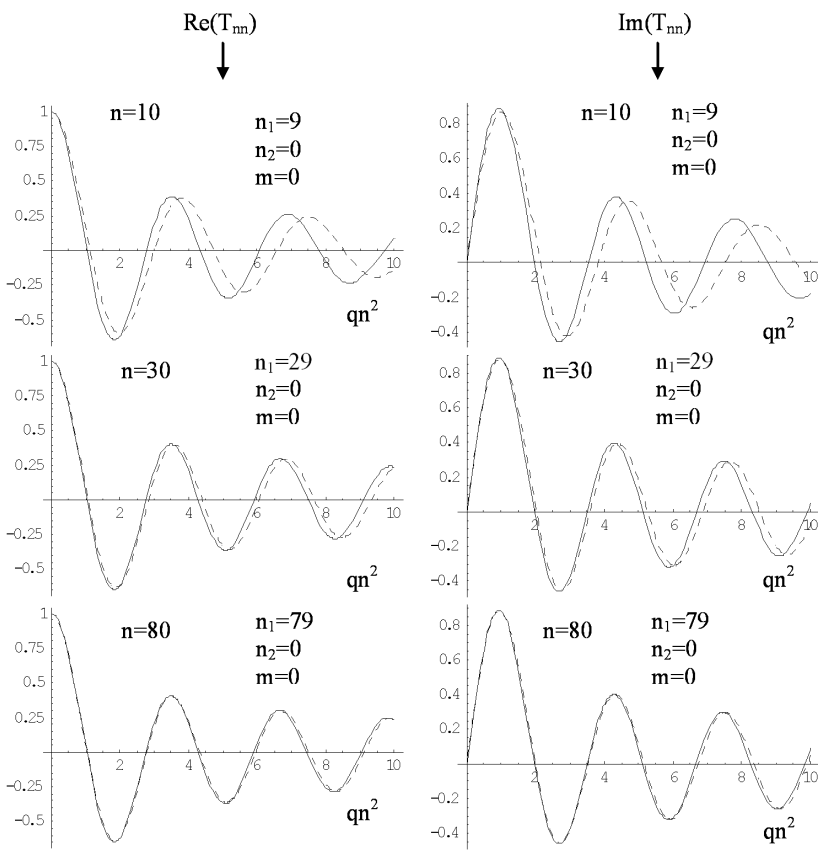

Fig. 1. Real (left) and imaginary (right) parts of the diagonal form factors of survival of the atom in its initial state versus the scaled momentum transferred to the atom. Solid line - the 1D approximation, dashed line - the full 3D results. The 3D calculations were performed for the extreme blue Stark states $\left(\left|n, n_{1}, n_{2},\right| m|\rangle=|10,9,0,0\rangle\right.$, $|30,29,0,0\rangle$, and $|80,79,0,0\rangle)$. 

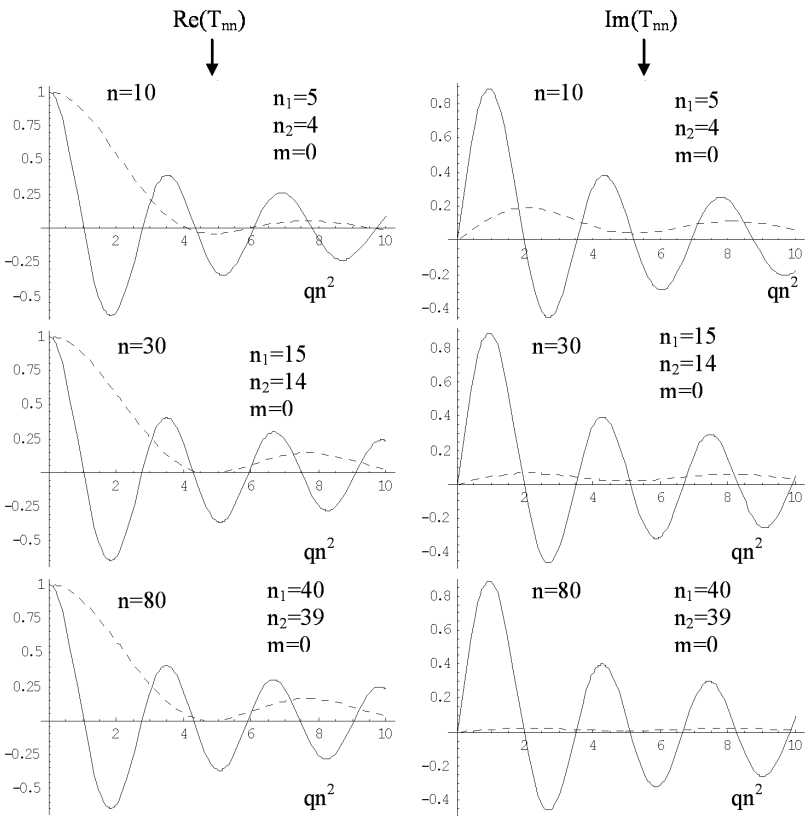

Fig. 2. As in Fig. 1 but for intermediate Stark states of a given $n$-manifold. Solid line — the $1 \mathrm{D}$ approximation, dashed line — the full 3D results.

Using the form factors (Eq. (6) and Eq. (8)) we can determine the free evolution of the wave packet after its excitation by HCP from the initial state $|n\rangle$;

$$
|\Psi(t)\rangle=\sum \mathrm{e}^{-\mathrm{i} E_{n^{\prime}} t} T_{n^{\prime}, n}|n\rangle,
$$

as well as the evolution of any expectation value for this wave packet. Specifically, we have investigated the evolution of the expectation value of position $x$ in both the 1D and 3D approaches. This expectation value is defined as $\langle x\rangle=\langle\Psi(t)|x| \Psi(t)\rangle=$ $\sum_{j j^{\prime}} T_{j^{\prime} i} T_{j i} x_{j^{\prime} j} \mathrm{e}^{\mathrm{i} \omega t}$ (see [10]), where the matrix element $x_{j^{\prime} j}$ is taken between two Rydberg states. These matrix elements can, formally, be obtained by calculating

$$
x_{\mathrm{fi}}=-\mathrm{i} \lim _{q \rightarrow 0} \frac{\partial T_{\mathrm{fi}}}{\partial q} .
$$

With the use of Eqs. (6) and (8), respectively, the 1D approximation gives

$$
x_{\mathrm{ii}}=\langle n|x| n\rangle=\frac{3}{2}(n)^{2}
$$

for the diagonal elements and

$$
\begin{aligned}
x_{\mathrm{fi}}= & \left\langle n^{\prime}|x| n\right\rangle=(-1)^{n} \frac{8 \sqrt{n n^{\prime}}\left(n n^{\prime}\right)^{2}}{\left(n^{\prime}-n^{\prime \prime}\right)^{4}}\left(\frac{n^{\prime}-n}{n^{\prime}+n}\right)^{n+n^{\prime}} \\
& \times\left\{\frac{2 n n^{\prime}-n-n^{\prime}}{n+n^{\prime}} F\left[1-n, 1-n^{\prime}, 2, z(y=0)\right]\right. \\
+ & \left.\frac{2 n n^{\prime}}{\left(n-n^{\prime}\right)^{2}}(n-1)\left(n^{\prime}-1\right) F\left[2-n, 2-n^{\prime}, 3, z(y=0)\right]\right\}
\end{aligned}
$$


for the off-diagonal elements [11]. In the 3D case we get [12]

$$
\begin{aligned}
& \quad x_{\mathrm{fi}}=-2(-1)^{n_{1}^{\prime}+n_{2}^{\prime}} \frac{1}{4(|m| !)^{2}} \sqrt{\frac{\left(n_{1}+|m|\right) !\left(n_{1}^{\prime}+|m|\right) !\left(n_{2}+|m|\right) !\left(n_{2}^{\prime}+|m|\right) !}{n_{1} ! n_{1}^{\prime} ! n_{2} ! n_{2}^{\prime} !}} \\
& \quad \times\left(\frac{4 n n^{\prime}}{\left(n-n^{\prime}\right)^{2}}\right)^{|m|+2}\left(\frac{n-n^{\prime}}{n+n^{\prime}}\right)^{n+n^{\prime}}\left\{\frac{2 n n^{\prime}\left(n_{1}-n_{2}\right)+\left(n_{2}^{\prime}-n_{1}^{\prime}\right)\left(n^{2}+n^{\prime 2}\right)}{\left(n+n^{\prime}\right)^{2}}\right. \\
& \quad \times F\left[-n_{1},-n_{1}^{\prime},|m|+1, z(y=0)\right] F\left[-n_{2},-n_{2}^{\prime},|m|+1, z(y=0)\right] \\
& \quad+n_{1}^{\prime} F\left[-n_{1},-n_{1}^{\prime}+1,|m|+1, z(y=0)\right] F\left[-n_{2},-n_{2}^{\prime},|m|+1, z(y=0)\right] \\
& \left.-n_{2}^{\prime} F\left[-n_{1},-n_{1}^{\prime},|m|+1, z(y=0)\right] F\left[-n_{2},-n_{2}^{\prime}+1,|m|+1, z(y=0)\right]\right\} .
\end{aligned}
$$

Applying the above formulae we have calculated the expectation value of $x$ for the Rydberg wave packet generated by a $\operatorname{HCP}\left(q=1.55 \times 10^{-3}\right.$ a.u. $)$ from the initial blue state of $n=25\left(n_{1}=24, n_{2}=0\right)$. This HCP redistributes the initial population over, mainly, the blue Stark states with $22 \leq n \leq 28$. The results are presented in Fig. 3, where we can see perfect agreement between the $1 \mathrm{D}$ and $3 \mathrm{D}$ approaches.

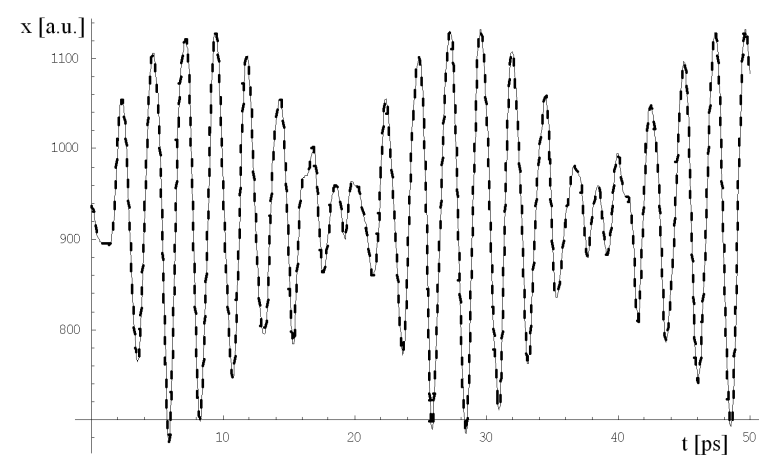

Fig. 3. Expectation value of the $x$ coordinate for a Rydberg wave packet as a function of time of free evolution. The wave packet was created by a HCP $\left(q=1.55 \times 10^{-3}\right.$ a.u. $)$ redistributing the initial population from the blue Stark state of $n=25$ to the manifold of seven blue Stark states with $22 \leq n \leq 28$. Dashed line - the $1 \mathrm{D}$ results, solid line - the $3 \mathrm{D}$ results.

Using the 1D approach we have, finally, calculated the evolution of localization of the wave packet by plotting squared modulus of Eq. (9) for different $t$. Since $|n\rangle$ is a polynomial of a finite order, the population should, for any $t$, oscillate along the $x$ axis, in general. For selected times of free evolution, Fig. 4 shows the distribution of population along the $x$ axis, obtained by the 1D and 3D approaches, for the wave packet consisting of the band of states $39 \leq n \leq 65$, 

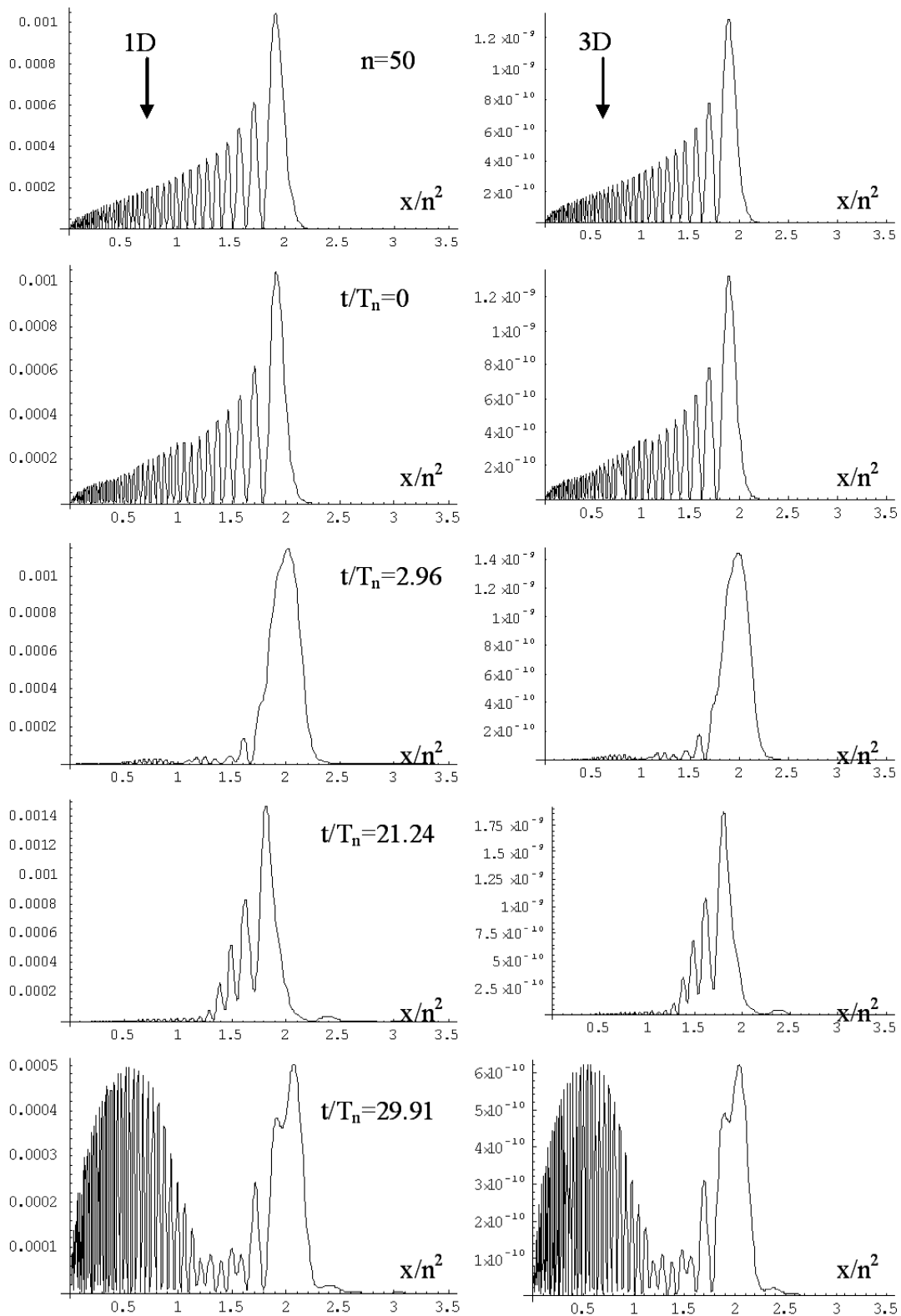

Fig. 4. The distribution along the $x$-axis for the freely evolving $1 \mathrm{D}$ (left column) and 3D (right column) wave packets produced by applying a HCP of $q=-0.001$ a.u. to the $n=50$ state. $t / T_{n}$ is the time of the evolution in units of the Kepler period for the initial state. On the vertical axis, the value of the squared modulus of the packet's wave function is given. 
excited from the initial state $|n\rangle=50$ by HCP of $q=-0.001$. With the use of Eq. (4) and the Fourier transform of Eq. (5) we also show in Fig. 5 the distribution in momentum space for the same wave packet. The agreement between the $1 \mathrm{D}$ and $3 \mathrm{D}$ results is very good as far as the shape is concerned, and different
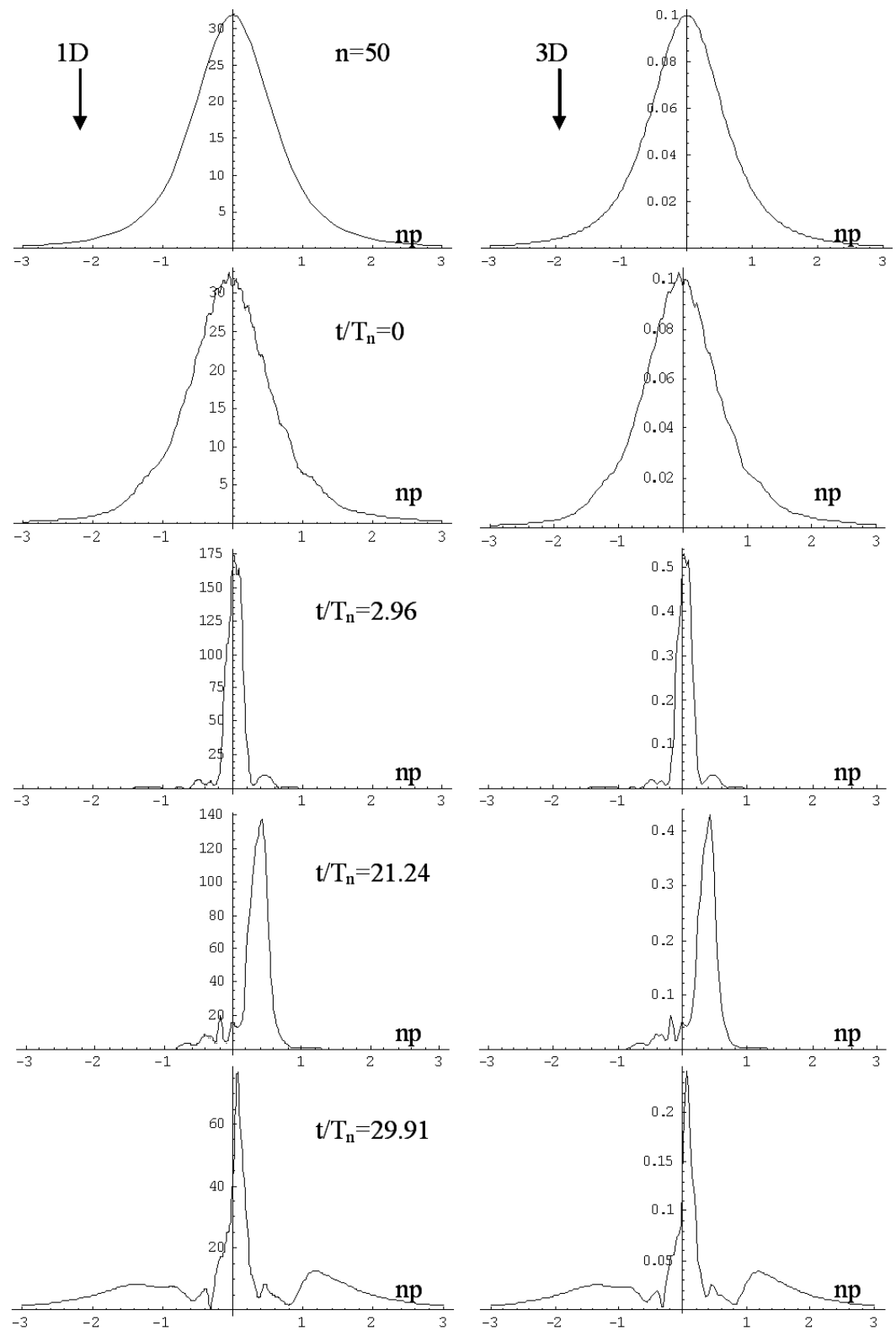

Fig. 5. The momentum distribution versus time of free evolution for the wave packet from Fig. 4 (left column - 1D results, right column - 3D results). On the vertical axis, the value of the squared modulus of the packet's wave function is given. 
heights of the 1D and 3D figures are caused by some spreading of the 3D wave function along other additional axis. The above choice of parameters is intentional in order to compare the present results with our recent ones [11] obtained along a different line, i.e., by calculating the position-momentum uncertainty product and the Husimi phase-space distribution function for the wave packet produced. For the specific times chosen, namely $t / T_{n}=2.96$ and $21.24\left(T_{n}\right.$ - the Kepler period of the initial $|n\rangle=50$ state) both the calculated uncertainty product and the Husimi function have suggested the best localization of the wave packet in the phase space. In contrary, for $t / T_{n}=29.91$ we have found in [11] the maximum delocalization of the wave packet in the phase space. The results of Fig. 4 and Fig. 5 confirm our previous results obtained by more sophisticated methods. To have a reference point, we have also included in Fig. 4 and Fig. 5 the results obtained for the initial state $|n\rangle=50$ and also for the wave packet just after its production, i.e., at $t / T_{n}=0$. Finally, we would like to stress that the two times of the best localization $\left(t / T_{n}=2.96\right.$ and 21.24$)$ are separated by the so-called revival time for the wave packet, i.e., by $n / 3 \approx 17$ in units of $T_{n}$.

\section{Summary}

All the results presented demonstrate that the real 3D Rydberg atom in its extreme Stark states of high $n \gg 1$ can be well approximated by its $1 \mathrm{D}$ counterpart. The 1D approach is simpler and more convenient in use and, when justified, offers results practically indistinguishable from the exact $3 \mathrm{D}$ ones.

\section{References}

[1] J. Ahn, T.C. Weinacht, P.H. Bucksbaum, Science 287, 463 (2000).

[2] R.R. Jones, D. You, P.H. Bucksbaum, Phys. Rev. Lett. 70, 1236 (1993).

[3] H. Maeda, T.F. Gallagher, Phys. Rev. Lett. 92, 133004 (2004).

[4] J. Bromage, C.R. Stroud Jr., Phys. Rev. Lett. 83, 4963 (1999).

[5] I. Bersons, R. Veilande, Phys. Rev. A 69, 043408 (2004).

[6] S.M. Susskind, R.V. Jensen, Phys. Rev. A 38, 711 (1988).

[7] L.D. Landau, E.M. Lifshitz, Quantum Mechanics, Pergamon, Oxford 1977.

[8] P. Krstic, Y. Hahn, Phys. Rev. A 48, 4515 (1993); Phys. Lett. A 192, 47 (1994).

[9] I. Bersons, A. Kulsh, Phys. Rev. A 55, 1674 (1997).

[10] R. Parzyński, M. Sobczak, Phys. Rev. A 65, 045401 (2002).

[11] R. Parzyński, T. Kopyciuk, M. Sobczak, Phys. Lett. A 352, 505 (2006).

[12] H.A. Bethe, E.E. Salpeter, Quantum Mechanics of One- and Two-Electron Atoms, Kluwer Academic, Dordrecht 1977. 